\title{
Announcement of Systematic Ionospheric Electron Density Data
}

Reduction of hourly ionospheric vertical soundings to electron density profiles has become a part of the systematic ionospheric data program of the Central Radio Propagation Laboratory, National Bureau of Standards. Scalings of ionograms for this purpose are being provided by ionosphere stations operated by CRPL and the U.S. Army Signal Corps. For the present, the hourly profile data from one CRPL station, Puerto Rico, are appearing in the monthly CRPL-F Reports, Part A. ${ }^{1}$ These data are in place of the standard ionogram reductions formerly provided by this station. The very considerable task of scaling the ionograms for this purpose is being undertaken by T. R. Gilliland, Engineer in Charge, Puerto Rico Ionosphere Sounding Station; the computations are performed at the NBS Boulder Laboratories by a group headed by J. W. Wright. Basic conversion of virtual to true heights uses the well-known matrix method developed by K. G. Budden of the Cavendish Laboratory, Cambridge University, programed for an IBM 650 computer.

The quantities given for each hour of each day of the month are: The electron density at each $10-\mathrm{km}$ interval of height, including the maximum electron density proportional to $\left(f_{0} F 2\right)^{2} ; h_{\text {min }}$, the height of zero or very low electron density, obtained by linear extrapolation of the $N(h)$ curve; $h_{\max }$, the height of maximum electron density, determined by fitting a parabola to the upper portion of the profile; and
$S\left(h_{\max }\right)$, the integrated electron density between $h_{\min }$ and $h_{\max }$.

Tabulations of two arithmetic mean electron densities are also given for each hour. One average is for the "undisturbed" ionosphere which includes the soundings taken when the magnetic character figure $K p$ is less than $4+$; the other includes the remaining data to form a "disturbed" average. The latter may have little physical significance because the number of disturbed hours is usually small and the behavior of the ionosphere during disturbed hours is not consistent.

Before the averaging process, the individual profiles are extrapolated above $h_{\max }$ by a Chapman distribution of $100-\mathrm{km}$ scale height. This assumed model seems to agree well with the few published measurements dealing with the topside profile of the $F$-region. Extrapolation is necessary in order to calculate homogeneous averages near $h_{\max }$ and the average profiles are, in fact, given up to $950 \mathrm{~km}$. Also given are the integrated electron densities estimated to infinity, $S\left(h_{\text {inf }}\right)$; this is an approximation to the total electron content in a vertical column of the ionosphere of $1 \mathrm{~cm}^{2}$ cross section.

1 The CRPL-F Reports, Part A, Ionospheric Data, have a limited press run and normally are available only on an exchange basis. However, compilations of tables of medians of ionospheric data and of tabulations of electron density appearing in this publication may be purcbased in booklet form at the price of appearing in this publication may be purcbased in booklet form at the price of $\$ 1.10$ per booklet. Please make inquiry of: IGY World Data Center A, Airglow
and Ionosphere, Central Radio Propagation Laboratory, National Bureau of and Ionosphere, Central
Standards, Boulder, Colo. 